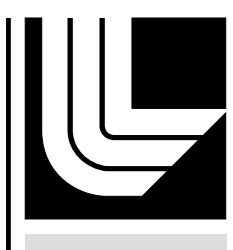

LAW RENCE LIVERMORE N A TIO N A L LABORATORY

\title{
UCRL-TR-218250
}

Keeping Cool Close to the Sun

A. Hazi

January 18, 2006 
This document was prepared as an account of work sponsored by an agency of the United States Government. Neither the United States Government nor the University of California nor any of their employees, makes any warranty, express or implied, or assumes any legal liability or responsibility for the accuracy, completeness, or usefulness of any information, apparatus, product, or process disclosed, or represents that its use would not infringe privately owned rights. Reference herein to any specific commercial product, process, or service by trade name, trademark, manufacturer, or otherwise, does not necessarily constitute or imply its endorsement, recommendation, or favoring by the United States Government or the University of California. The views and opinions of authors expressed herein do not necessarily state or reflect those of the United States Government or the University of California, and shall not be used for advertising or product endorsement purposes.

This work was performed under the auspices of the U.S. Department of Energy by University of California, Lawrence Livermore National Laboratory under Contract W-7405-Eng-48. 


\section{Keeping Cool Close to the Sun}

$T^{+}$ HE germanium detector in the gamma-ray spectrometer (GRS)

aboard the MESSENGER spacecraft is only the size and weight of a can of peaches but will play a critical role in investigating Mercury, the planet closest to the Sun. The MESSENGER (MErcury Surface, Space ENvironment, GEochemistry, and Ranging) spacecraft travels at about 38 kilometers per second and is named after the scientific goals of the mission. It is the first spacecraft to visit Mercury since 1975.

MESSENGER must take an oblique route to approach Mercury so that it does not fly past the planet and fall directly into the Sun. The

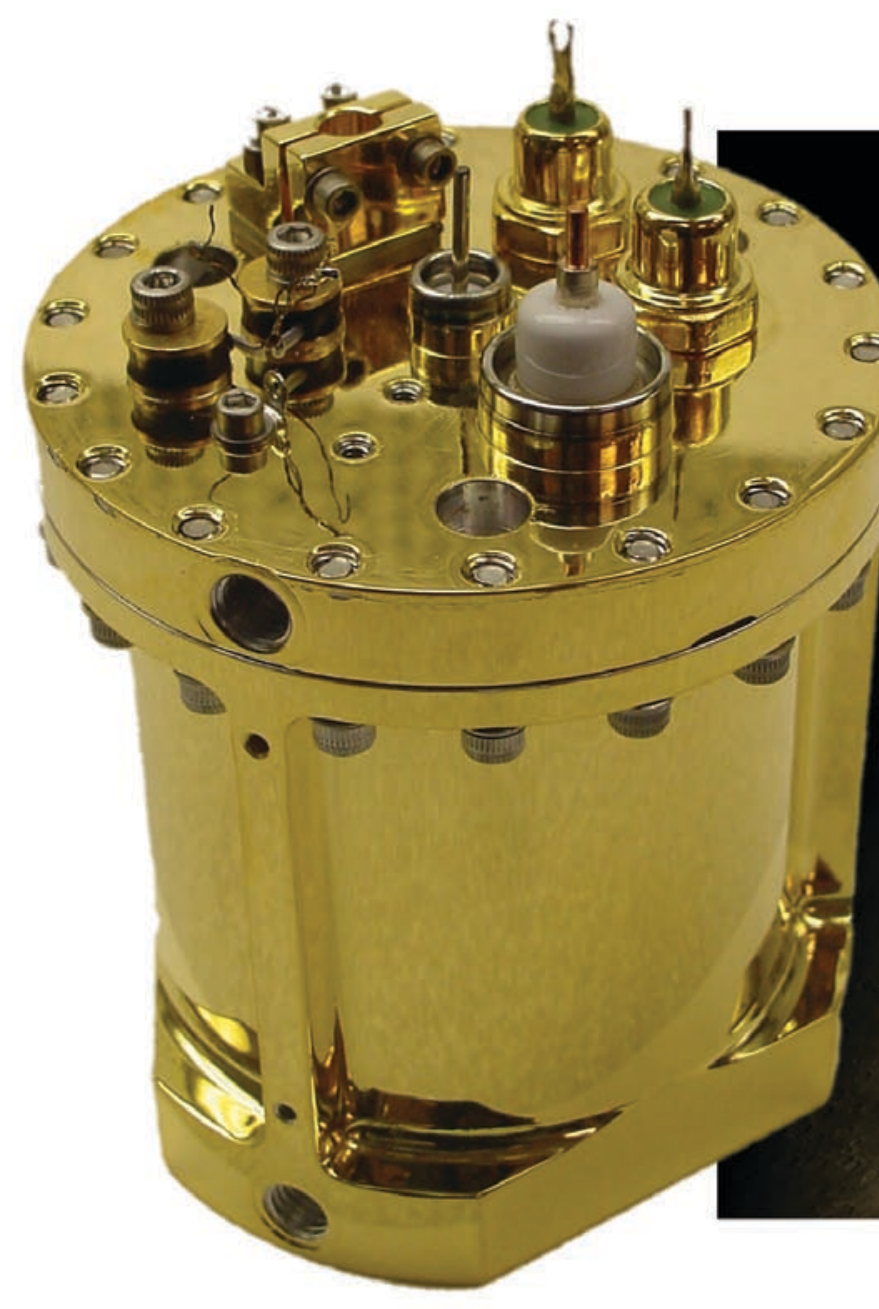

spacecraft will travel 7.9 billion kilometers, flying by Earth once, Venus twice, and Mercury three times before settling into orbit around this mysterious planet. Of all the terrestrial planets, which include Venus, Earth, and Mars, Mercury is the smallest and the densest; its days are 176 Earth days long, two complete orbits of the planet around the Sun. Temperatures range from a high of $450^{\circ} \mathrm{C}$ on the Sun side during its long day to a low of $-185^{\circ} \mathrm{C}$ on its night side. By studying this extreme planet, scientists hope to better understand how Earth formed and evolved.

The GRS, one of the seven lightweight scientific instruments on MESSENGER, will be used to help scientists determine the abundance of elements in Mercury's crust, including the materials that might be ice at its poles. Livermore engineer Norman Madden 
led the West Coast team effort to design and build the GRS in a collaboration led by Johns Hopkins University Applied Physics Laboratory (JHUAPL). The team included Lawrence Berkeley and Lawrence Livermore national laboratories as well as University of California at Berkeley (UCB) Space Sciences Laboratory (SSL). The JHUAPL MESSENGER project is a National Aeronautics and Space Administration (NASA) Discovery Mission.

\section{Detecting the Elements in Mercury's Surface}

At the heart of the GRS is a germanium detector that will measure the gamma rays coming from Mercury's surface. Mercury is constantly bombarded with cosmic rays, and because it has almost no atmosphere, the planet's surface is activated and gives off gamma rays that are detectable from orbit. But not all gamma rays are alike. The energy of each gamma ray varies, depending on the type of element it comes from.

The germanium detector consists of a 5-centimeter-diameter, 5-centimeter-long germanium crystal that is electromechanically cooled and encapsulated in a highly reflective gold-plated container with a pressurized, ultrapure nitrogen-gas atmosphere. When illuminated by hard $\mathrm{x}$ rays or gamma rays, the germanium crystal releases electron hole pairs, which create a current as they are pulled to the inner and outer electrodes of the detector. This current pulse, which is proportional to the incident gamma-ray energy, is amplified and converted into digital data.

The data are accumulated and eventually compared to a catalog of element signatures. These signature gamma rays enable researchers to determine the elements that make up Mercury's surface. By counting the number of each type of gamma ray, researchers will also be able to determine the relative abundance of the elements.

Scientists have been puzzled by certain deposits in craters at Mercury's poles. Although most of Mercury experiences extreme heat during its long days, portions of these craters never get direct sunlight, and scientists wonder if the reflective material detected there could be ice. With the GRS data, scientists could determine whether these materials contain hydrogen in water ice or whether the deposits are formed of sulfur or some other element.

With its high energy resolution, the germanium detector obtains precise information from the planet's surface while it remains in space_-but doing so is a challenge. The detector must be cooled to a very low temperature (about $-185^{\circ} \mathrm{C}$ ), and it must survive solar flares and repair itself when it accumulates radiation damage.

Bombardment by energetic particles from the Sun and outer space (mostly high-energy protons) damages the germanium crystal and degrades the energy resolution. Damage is repaired by a process called annealing; the temperature of the crystal is slowly raised to $85^{\circ} \mathrm{C}$ and then sustained for a few days before being lowered back down to $-185^{\circ} \mathrm{C}$.

\section{Keeping It Cool}

Because the detector needs to operate at very low temperatures and MESSENGER is close to the Sun, the thermal design to protect the detector was critical. The detector is kept cool by an electromechanical cryocooler attached to the outside of the device. However, the cryocooler has a limited cooling capacity because of size and weight constraints.

To ensure the cryocooler would sufficiently cool the detector, Livermore scientists used SINDA/FLUINT, a commercial program originally developed by NASA, to model the thermal environments that the spectrometer was expected to encounter-during liftoff, in space while en route to Mercury, and in orbit around the planet. Using the data from the model, scientists from Lawrence Livermore and Lawrence Berkeley developed a design that included three closely spaced and highly reflective thermal shields held in place with DuPont KEVLAR® fiber.

\section{Assembly Challenges}

Assembling the detector, its heat shields, and its external cage was particularly challenging. All the pieces had to be held in place, with all the required gaps between them, while the KEVLAR ${ }^{\circledR}$ was strung in two directions around the cage under tension. Once assembled, the entire device had to be held in place while the epoxy glue holding the ends of the fiber cured over 24 hours.

When designing the assembly, the team found an article by Pat Roach at NASA Ames about using KEVLAR ${ }^{\circledR}$ to hold a cryogenic device in place and determined that particular fiber would also be appropriate for the GRS. The advantages of KEVLAR $®$ are that it is very strong, has low heat conductivity, and does not outgas. However, during testing, the team did discover that the fiber frays. The team encountered problems when tying off the ends, and the fiber tended to fray when it was bent or in contact with a metal edge.

"We solved the fraying problem by widening the radius of the pegs and removing any sharp edges that were touched by the fiber," says Livermore engineer Monika Witte. "And instead of tying off the ends, we used an epoxy to glue the ends in place." This epoxy needed to meet NASA's specifications to ensure that no fumes from the epoxy could contaminate the detector or other scientific equipment on MESSENGER.

Once assembled, the device was tested to ensure that the acceleration and vibration during launching would not affect the fiber rigging, cryogenic cold finger, wire connections, or gaps between the detector, thermal shields, and external cage. Livermore performed sinusoidal and random vibration tests, during which the device was mounted on a shaker table and vibrated with constant amplitude through a range of frequencies. The test frequencies represented the full range of required test conditions without 


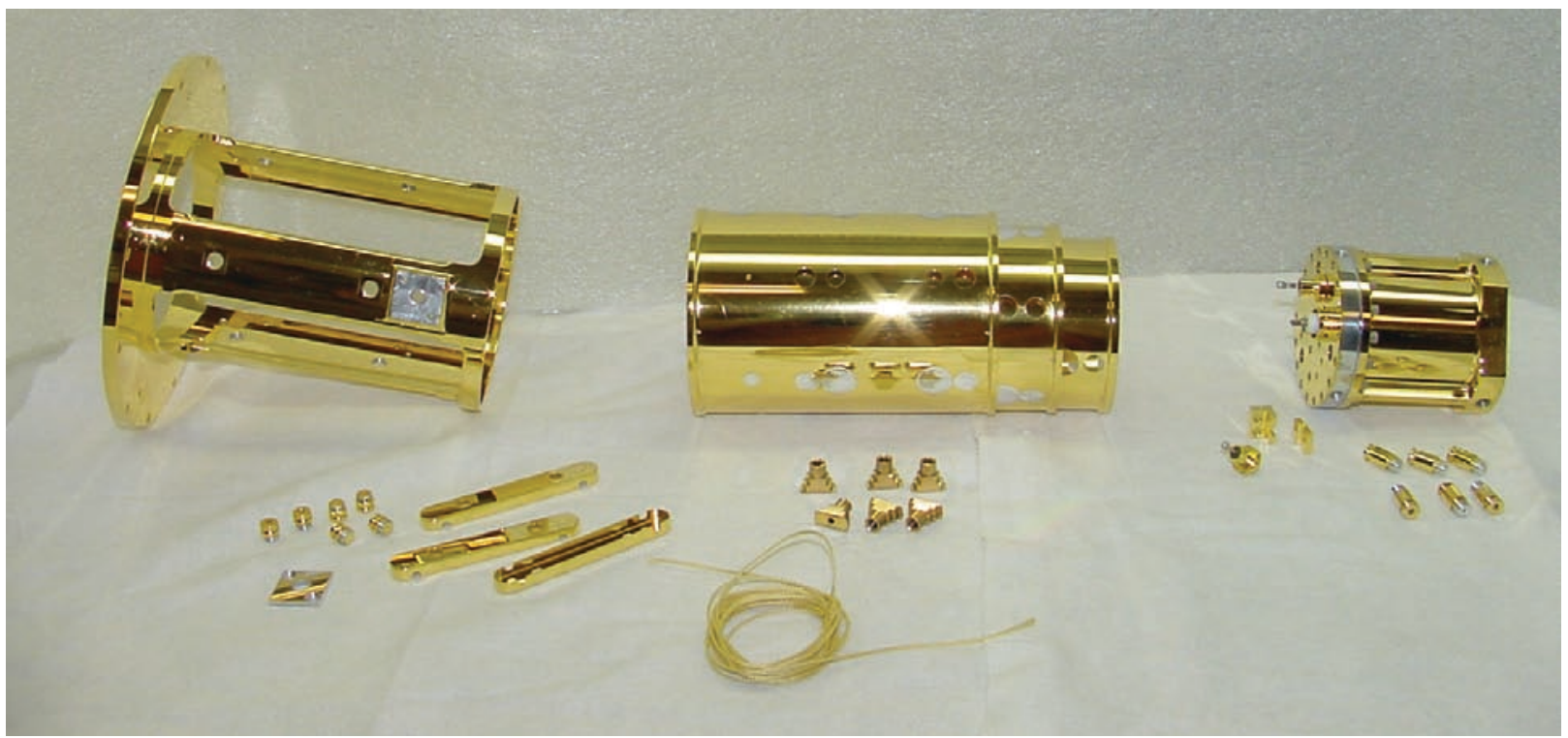

All parts of the germanium detector assembly (right), its thermal shielding (center), and its external cage (left) are highly polished and gold-plated to minimize radiativeheat transfer.

exceeding limits and overstressing the instrument. The tests showed that the external cage was stiff enough to minimize disjointed vibration (where two parts of the GRS are vibrating at different rates) and flexible enough not to break under the strain.

\section{The Best Kind of Collaboration}

"This was truly a collaborative effort," says Madden. "All the team members had to work very closely and cooperatively together to make this project a success." The detector was designed and built by Lawrence Berkeley for JHUAPL. Lawrence Livermore performed the thermal analysis as well as the vibration analysis and testing. UCB's SSL fabricated the many spacequalified mechanical components. Lawrence Berkeley and Lawrence Livermore collaborated with SSL in assembling the detector, thermal shields, and cage device in a space-certified clean room.

\section{Early Results}

Launched on August 3, 2004, MESSENGER is on its six-anda-half-year trip to Mercury. While traveling, the GRS is already recording gamma rays from space and with slightly better resolution than expected. In addition, the detector has undergone a scheduled anneal cycle. Scientists are excited about these promising early results and look forward to the data the GRS will obtain once in orbit around Mercury.

- Karen Rath

Key Words: gamma-ray spectrometer (GRS), germanium detector, KEVLAR ${ }^{\circledR}$ fiber, Mercury, MESSENGER, thermal shielding, vibration testing.

\section{For further information contact Norman Madden (925) 423-1934, (madden8@IInl.gov).}

